\title{
Simulação da Dispersão de Poluentes na Atmosfera com Deposição Seca no Solo
}

\author{
Juliana Contreira $^{1, *}$ Daniela Buske $^{2} \quad$ Régis Quadros $^{2}$ Glênio Gonçalves ${ }^{2}$ \\ ${ }^{1}$ Graduanda em Engenharia Civil, UFPel \\ ${ }^{2}$ Depto de Matemática e Estatística, IFM, UFPel \\ 96010-610, Pelotas, RS \\ E-mail: julianacontreira@hotmail.com, daniela.buske@ufpel.edu.br, \\ regis.quadros@ufpel.edu.br, gleniogoncalves@yahoo.com.br
}

\section{RESUMO}

Este trabalho apresenta uma solução analítica para a equação de advecção-difusão-deposição bidimensional, considerando a deposição seca no solo. Esta solução é utilizada para simular a dispersão de poluentes na baixa atmosfera e, não tem nenhuma limitação quanto ao coeficiente de difusão turbulenta e perfil de vento dependentes da altura. Para a obtenção dos campos de concentração, são utilizados os dados do experimento difusivo realizado em Hanford (1983).

Considera-se a equação de advecção-difusão-deposição bidimensional transiente com deposição seca no solo, escrita na seguinte forma [4][5]:

$$
\frac{\partial c}{\partial t}+u \frac{\partial c}{\partial x}=\frac{\partial}{\partial z}\left(K_{Z} \frac{\partial c}{\partial z}\right)
$$

onde $x$ é a direção do vento médio, $c$ é a concentração integrada em $y\left(\mathrm{~g} / \mathrm{m}^{2}\right), u$ é o vento médio, $K_{z}$ é o coeficiente de difusão turbulento dependente da altura $z$. A Eq. (1) está as seguintes condições de contorno, de fonte e inicial:

$$
\begin{gathered}
K_{z} \frac{\partial c(x, h, t)}{\partial z}=0 \quad \text { em } z=h \\
K_{z} \frac{\partial c\left(x, z_{0}, t\right)}{\partial z}=V_{d} c \quad \text { em } z=z_{0} \\
u c(0, z, t)=Q \delta\left(z-H_{s}\right) \quad \text { em } x=0 \\
c(x, z, 0)=0 \quad \text { quando } t=0
\end{gathered}
$$

onde $V_{d}$ é a velocidade de deposição no nível do solo, $Q$ é a taxa de emissão do poluente, $H_{s}$ é a altura da fonte, $\delta$ é a função delta de Dirac, $z_{0}$ é a rugosidade do terreno e $h$ é a altura da camada limite atmosférica.

O problema proposto foi resolvido com o uso das técnicas da transformada de Laplace e GILTT (Generalized Integral Laplace Transform Technique) [4][5]. A concentração do poluente é expandida em função de uma base de autofunções, advinda de um problema de Sturm-Liouville, na seguinte forma:

$$
c(x, z, r)=\sum_{n=0}^{\infty} c_{n}(x, r) \psi_{n}(z)
$$

onde $\psi_{n}(z)$ é a autofunção $\psi_{n}(z)=\cos \lambda_{n}(z-h)$ e $\lambda_{n}$ é o autovalor obtido ao se resolver a equação transcendental $V_{d} / K_{z}=\lambda_{n} \tan \left(\lambda_{n}\left(h-z_{0}\right)\right)$. Uma vez encontrados os valores de $C_{n}(x, r) \mathrm{o}$ problema estará solucionado.

Substituindo a Eq. (2) no problema original, aplicando o operador integral $\int_{0}^{h}(.) \psi_{m} d z$ onde $\psi_{m}$ é uma função ortogonal à $\psi_{n}$, a equação resultante reescrita em notação matricial é dada por:

$$
Y^{\prime}(x, r)+F . Y(x, r)=0
$$

$\mathrm{A}$ matriz $\mathrm{F}$ é dada por $\mathrm{F}=\mathrm{A}^{-1} \mathrm{~B}$, onde os termos de $\mathrm{A}$ e $\mathrm{B}$ são definidos como:

$$
a_{n m}=\left\{\int u \psi_{n} \psi_{m} d z\right\} \text { e } b_{n m}=\left\{-\int K_{z}^{\prime} \psi_{n}^{\prime} \psi_{m} d z+\lambda_{n}{ }^{2} \int K_{z} \psi_{n} \psi_{m} d z+r \int \psi_{n} \psi_{m} d z\right\}
$$

O problema transformado (3) é resolvido aplicando a transformada de Laplace na variável $x \mathrm{e}$ diagonalização da matriz F. Finalmente, aplicando a transformada inversa de Laplace no tempo teremos a solução do problema transiente totalmente determinada. Esta é obtida numericamente pelo método de inversão numérica da quadratura Gaussiana:

$$
\mathrm{c}(\mathrm{x}, \mathrm{z}, \mathrm{t})=\sum_{\mathrm{k}=1}^{\mathrm{M}} \frac{\mathrm{P}_{\mathrm{k}}}{\mathrm{t}} \mathrm{A}_{\mathrm{k}} \sum_{\mathrm{n}=0}^{\mathrm{N}} \mathrm{c}_{\mathrm{n}}\left(\mathrm{x}, \frac{\mathrm{P}_{\mathrm{k}}}{\mathrm{t}}\right) \psi_{n}(\mathrm{z})
$$

onde $A_{k}$ e $P_{k}$ são os pesos e raízes da quadratura.

Uma vez obtida a solução, é necessário selecionar o perfil de vento e o coeficiente de difusão vertical. A partir de um ponto de vista físico, uma parametrização da turbulência é uma aproximação da natureza no sentido que os modelos matemáticos recebem uma relação aproximada

* Bolsista de Iniciação Científica PIBIC/CNPq 
que substitui um termo desconhecido. O coeficiente de difusão turbulenta usado é dado por Degrazia et. al. [2], sendo este um coeficiente que leva em conta a turbulência do fenômeno de uma forma mais completa do que o utilizado em [5][1]. O perfil de vento utilizado é descrito por uma lei de potencia. Como um exemplo de aplicação da solução obtida, utilizou-se o conjunto de dados do experimento difusivo de Hanford [3].

$\mathrm{Na}$ figura 1 é apresentado o gráfico de espalhamento entre os dados medidos experimentalmente e os preditos pela GILTT ao nível do solo para os diferentes perfis de vento. O modelo foi avaliado com a razão $C_{d} / C_{n d}$, onde $C_{d}$ e $C_{n d}$ são as concentrações do $Z_{n} S$ e $S_{6}$ medidas a 1,5m acima do solo (índice $d$ significa deposita e índice $n d$ significa não-deposita). Podemos observar uma boa concordância entre os resultados obtidos pela GILTT e os dados experimentais. Os resultados obtidos apresentam uma melhora estatística em relação aos obtidos em [5], mostrando que a escolha do coeficiente de difusão turbulenta foi mais adequada.

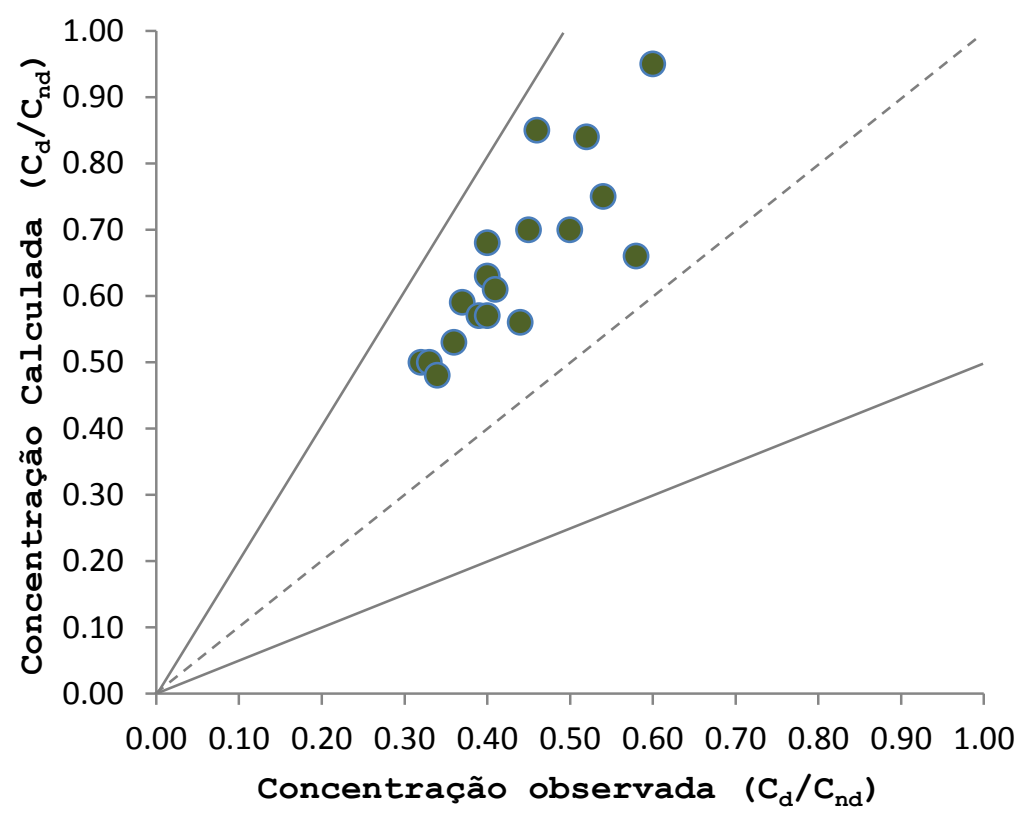

Figura 1: Diagrama de espalhamento dos dados preditos $\left(C_{p}\right)$ pela GILTT em comparação com os dados observados $\left(C_{o}\right)$ experimentalmente. Dados entre as linhas cheias estão dentro do fator de dois $\left(0,5 \leq \frac{C_{p}}{C_{o}} \leq 2\right)$.

Palavras-chave: solução analítica, equação de advecção-difusão-deposição, método espectral

\section{Referências}

[1]G. A. Degrazia, H.F.Campos Velho, J.C. Carvalho, Nonlocal exchange coefficients for the convective boundary-layer derived from spectral properties, C.Atmos.Physics, pp. 57-64. (1997).

[2]G. A. Degrazia, D. Anfonssi, J.C. Carvalho, C.Mangia, T. Tirabassi, H.F.Campos Velho, Turbulence parametrisation for PBL dispersion models in all stability conditions, Atmos. Environ., vol. 30, pp. 3575-3583. (2000).

[3]J.C. Doran, O.B. Abbey, J.W. Buck, D.W. Glover, T.W. Horst, Field validation of Exposure Assessment Models. Data Environmental Science Research Lab, Res. Triangle Park: NC. (1984).

[4]D.M. Moreira, M.T. Vilhena, D. Buske, T. Tirabassi, The state-of-art of the GILTT method to simulate pollutant dispersion in the atmosphere. Atmos. Research, vol. 92, pp. 117, (2009).

[5]D.A. Schuch, G.J. Weymar, I. Furtado, R.S. Quadros, D. Buske, Simulação da dispersão de poluentes na camada limite atmosférica incluindo o efeito de deposição seca no solo. Ciencia e Natura (2011). 